\title{
cis-Dioxomolybdenum(VI) and Oxo(phosphine
}

\section{oxide)molybdenum(IV) Complexes: Steric and Electronic Fine- Tuning of cis-[MoOS $]^{2+}$ Precursors}

Christian J. Doonan, Andrew J. Millar, David J. Nielsen and Charles G. Young*

School of Chemistry, University of Melbourne, Victoria 3010, Australia

\section{Supporting Information}

Table 1. Microanalytical data and mass spectrometric data for $\mathrm{Tp}^{\mathrm{iPr}} \mathrm{MoO}_{2}(\mathrm{OAr})$ complexes.

Table 2. Microanalytical and mass spectrometric data for $\mathrm{Tp}^{\mathrm{iPr}} \mathrm{MoO}(\mathrm{OAr})\left(\mathrm{OPEt}_{3}\right)$ complexes.

Listing 1. Full listings of IR bands for new compounds (in $\mathrm{KBr}$ ). 
Table 1. Microanalytical data and mass spectrometric data for $\mathrm{Tp}^{\mathrm{iPr}} \mathrm{MoO}_{2}(\mathrm{OAr})$ complexes. ${ }^{\mathrm{a}}$

\begin{tabular}{lcccc}
\hline OAr ligand & $\mathrm{C}$ & $\mathrm{H}$ & $\mathrm{N}$ & {$[\mathrm{M}+\mathrm{H}]^{+} \mathrm{m} / z$} \\
& \% obs (calc) & \% obs (calc) & \% obs (calc) & \\
\hline $\mathrm{OC}_{6} \mathrm{H}_{4}{ }^{\mathrm{t}} \mathrm{Bu}-2(\mathbf{1})$ & $54.13(54.56)$ & $6.74(6.70)$ & $13.56(13.63)$ & 619.4 \\
$\mathrm{OC}_{6} \mathrm{H}_{3} \mathrm{Me}_{2}-2,6(\mathbf{3})$ & $51.82(53.07)$ & $6.26(6.34)$ & $14.45(14.29)$ & 591.4 \\
$\mathrm{OC}_{10} \mathrm{H}_{7}-\alpha(\mathbf{4})$ & $55.24(55.10)$ & $5.74(5.78)$ & $13.95(13.77)$ & 613.4 \\
$\mathrm{OC}_{6} \mathrm{H}_{4} \mathrm{Ph}-2(\mathbf{5})$ & $55.73(55.62)$ & $5.91(5.86)$ & $12.96(13.21)$ & 639.4 \\
$\mathrm{OC}_{6} \mathrm{H}_{4} \mathrm{Ph}-3(\mathbf{6})$ & $55.75(55.62)$ & $5.90(5.86)$ & $13.05(13.21)$ & 639.4 \\
$\mathrm{OC}_{10} \mathrm{H}_{7}-\beta(\mathbf{9})$ & $55.10(55.10)$ & $5.78(5.78)$ & $13.73(13.77)$ & 613.4 \\
$\mathrm{OC}_{6} \mathrm{H}_{4} \mathrm{Ph}-4(\mathbf{1 0})$ & $56.47(56.62)$ & $5.95(5.86)$ & $13.24(13.21)$ & 639.4 \\
$\mathrm{OC}_{6} \mathrm{H}_{4}{ }^{\mathrm{t}} \mathrm{Bu}-4(\mathbf{1 1})$ & $54.53(54.56)$ & $6.71(6.70)$ & $13.75(13.63)$ & 619.4 \\
$\mathrm{OC}_{6} \mathrm{H}_{4}{ }^{\mathrm{s}} \mathrm{Bu}-4(\mathbf{1 2})$ & $54.43(54.56)$ & $6.71(6.70)$ & $13.73(13.63)$ & 619.4 \\
\hline
\end{tabular}

${ }^{\mathrm{a}}$ For data for 2, 7 and 8, see Millar et al., Inorg. Chim. Acta 2002, 337, 393. 
Table 2. Microanalytical and mass spectrometric data for $\mathrm{Tp}^{\mathrm{iPr}} \mathrm{MoO}(\mathrm{OAr})\left(\mathrm{OPEt}_{3}\right)$ complexes.

\begin{tabular}{|c|c|c|c|c|}
\hline OAr Ligand & $\mathrm{C}$ & $\mathrm{H}$ & $\mathrm{N}$ & {$[\mathrm{M}]^{+} \mathrm{m} / z$} \\
\hline & $\%$ obs (calc) & $\%$ obs (calc) & $\%$ obs (calc) & \\
\hline $\mathrm{OC}_{6} \mathrm{H}_{4}{ }^{\mathrm{t}} \mathrm{Bu}-2$ & $55.45(55.59)$ & $7.56(7.68)$ & $11.30(11.43)$ & 736.3 \\
\hline $\mathrm{OC}_{10} \mathrm{H}_{7}-\alpha$ & $56.38(56.05)$ & $6.96(6.92)$ & $11.54(11.80)$ & 730.3 \\
\hline $\mathrm{OC}_{6} \mathrm{H}_{4} \mathrm{Ph}-2$ & $57.00(57.30)$ & $7.01(6.95)$ & 11.09 (11.14) & 756.3 \\
\hline $\mathrm{OC}_{6} \mathrm{H}_{4} \mathrm{Ph}-3$ & $57.02(57.30)$ & $6.95(6.95)$ & $11.06(11.14)$ & 756.3 \\
\hline $\mathrm{OC}_{6} \mathrm{H}_{4}{ }^{\mathrm{t}} \mathrm{Bu}-3$ & $55.49(55.59)$ & 7.77 (7.68) & $11.36(11.43)$ & 736.3 \\
\hline $\mathrm{OC}_{6} \mathrm{H}_{3}{ }^{\mathrm{t}} \mathrm{Bu}_{2}-3,5$ & $57.49(57.68)$ & $8.13(8.16)$ & $10.39(10.62)$ & 792.3 \\
\hline $\mathrm{OC}_{10} \mathrm{H}_{7}-\beta$ & $56.50(56.05)$ & $6.99(6.92)$ & $11.70(11.80)$ & 730.3 \\
\hline $\mathrm{OC}_{6} \mathrm{H}_{4} \mathrm{Ph}-4$ & $56.99(57.30)$ & $6.90(6.95)$ & $11.05(11.14)$ & 756.3 \\
\hline $\mathrm{OC}_{6} \mathrm{H}_{4}{ }^{\mathrm{t}} \mathrm{Bu}-4$ & $55.21(55.59)$ & 7.63 (7.68) & $11.29(11.43)$ & 736.3 \\
\hline $\mathrm{OC}_{6} \mathrm{H}_{4}{ }^{\mathrm{s}} \mathrm{Bu}-4$ & $55.66(55.59)$ & $7.74(7.68)$ & $11.41(11.44)$ & 736.3 \\
\hline
\end{tabular}

${ }^{\mathrm{a}}$ For analysis of $\mathrm{Tp}^{\mathrm{iPr}} \mathrm{MoO}(\mathrm{OPh})\left(\mathrm{OPEt}_{3}\right)$, see Smith et al. J. Am. Chem. Soc. 2000, 122, 9298 and for the $\mathrm{X}=\mathrm{OC}_{6} \mathrm{H}_{4}{ }^{\mathrm{s}} \mathrm{Bu}-2, \mathrm{OC}_{6} \mathrm{H}_{4}{ }^{\mathrm{t}} \mathrm{Bu}-3$ and $\mathrm{OC}_{6} \mathrm{H}_{3}{ }^{\mathrm{t}} \mathrm{Bu}_{2}-3,5$ derivatives, see Millar et al. Chem. Eur. J. 2005, in press. 
Listing 1. Full listings of IR bands for new compounds (in $\mathrm{KBr}$ ). ${ }^{\mathrm{a}}$

\section{Dioxo-Mo(VI) Complexes}

$\mathrm{Tp}^{\mathrm{iPr}} \mathrm{MoO}_{2}\left(\mathrm{OC}_{6} \mathrm{H}_{4}{ }^{\mathrm{B}} \mathrm{Bu}-2\right)$ (1): 3109 w, 2967 s, 2955 m, 2929 m, 2907 m, 2869 m, 2477 m, 2456 m, 1591 m, 1569 w, 1507 s, 1481 m, 1460 m, 1438 m, 1403 m, 1387 m, 1363 m, 1291 w, 1274 sh w, 1256 s, 1192 m, 1161 w, 1161 w, 1106 w, 1091 m, 1083 m, 1072 m, 1062 m, 1047 s, 1025 w, 931 s, 903 s, 835 w, 820 w, 796m, 781 m, 750 m, 737 m, 730 m, $714 \mathrm{w}, 636 \mathrm{~m}, 566 \mathrm{w}, 492 \mathrm{w}, 435 \mathrm{w}, 424 \mathrm{w} \mathrm{cm}^{-1}$.

$\mathrm{Tp}^{\mathrm{iPr}} \mathrm{MoO}_{2}\left(\mathrm{OC}_{6} \mathrm{H}_{3}-\mathrm{Me}_{2}-2,6\right)(3): 3135$ w, 3119 w, 2969 m, 2925 m, 2870 m, 2487 m, 2461 w, 1753 w, 1589 w, 1508 s, 1492 m, 1469 w, 1440 w, 1400 m, 1385 m, 1363 m, 1292 w, 1270 m, 1223 s, 1195 s, 1162 w, 1105 m, 1083 m, 1072 m, 1062 w, 1049 m, 935 s, 907 s, 895 s, 819 w, 797 m, 778 m, 758 m, 742 sh w, 732 s, 627 m, 564 m, 546 w, 427 w cm ${ }^{-1}$. $\mathrm{Tp}^{\mathrm{iPr}} \mathrm{MoO}_{2}\left(\mathrm{OC}_{10} \mathrm{H}_{7}-\alpha\right)(4): 3143$ w, 3123 w, 3109 w, 3055 w, 2970 m, 2928 m, 2870 m, 2505 m, 1589 sh, 1573 m, 1507 s, 1460 m, 1445 sh, 1388 s, 1365 m, 1325 w, 1295 m, 1268 m, 1238 m, 1198 s, 1171 sh, 1155 sh, 1090 m, 1071 m, 1050 s, 1023 m, 961 w, 928 s, 905 s, 820 w, 794 m, 770 m, 732 m, 715 sh, 658 w, 639 m, 570 w, 549 sh, 511 w, 473 $\mathrm{w}, 423 \mathrm{w} \mathrm{cm}^{-1}$.

$\mathrm{Tp}^{\mathrm{iPr}} \mathrm{MoO}_{2}\left(\mathrm{OC}_{6} \mathrm{H}_{4} \mathrm{Ph}-2\right)(5): 3141$ w, 3063 w, 2971 m, 2928 m, 2869 m, 2502 m, 1593 w, 1507 s, 1473 m, 1462 w, 1427 m, 1401 m, 1385 m, 1364 m, 1291 w, 1261 w, 1241 w, 1226 m, 1197 m, 1161 w, 1115 w, 1106 w, 1081 sh, 1072 m, 1064 sh, 1050 s, 1027 w, 1021 w, 1009 sh, 937 s, 905 s, 874 m, 820 w, 797 m, 784 m, 774 sh, 752 m, 733 s, 714 sh, 697 w, 657 w, 638 m, 610 w, 528 w, 493 w, 469 w, $423 \mathrm{w} \mathrm{cm}^{-1}$.

$\mathrm{Tp}^{\mathrm{iPr}} \mathrm{MoO}_{2}\left(\mathrm{OC}_{6} \mathrm{H}_{4} \mathrm{Ph}-3\right)$ (6): 3435 br, 3062 w, 2969 m, 2927 m, 2869 m, 2494 m, 2460 m, 1589 m, 1566 m, 1507 s, 1471 m, 1400 m, 1385 m, 1364 m, 1297 m, 1199 s, 1160 m, 1104 m, 1082 m, 1070 m, 1047 s, 1027 m, 997 w, 931 m, 900 s, 820 w, 797 m, 786 m, 774 m, 757 m, 749 m, 732 m, 698 m, 644 m, 610 m, 492 w, 480 w, $423 \mathrm{w} \mathrm{cm}^{-1}$.

$\mathrm{Tp}^{\mathrm{iPr}} \mathrm{MoO}_{2}\left(\mathrm{OC}_{10} \mathrm{H}_{7}-\beta\right)(9): 3119$ w, 3055 w, 2970 m, 2929 m, 2870 m, 2545 m, 2493 m, 1738 w, 1626 m, 1593 m, 1505 s, 1462 s, 1402 m, 1386 m, 1365 m, 1295 m, 1255 s, 1218 m, 1196 m, 1175 m, 1122 w, 1106 w, 1080 w, 1070 w, 1051 m, 1024 w, 965 m, 924 s, 903 s, 849 m, 811 w, 782 m, 743 sh, 732 m, 626 m, 567 m, 514 w, 494 w, 475 w, 453 w, 424 $\mathrm{cm}^{-1}$. 
$\mathrm{Tp}^{\mathrm{iPr}} \mathrm{MoO}_{2}\left(\mathrm{OC}_{6} \mathrm{H}_{4} \mathrm{Ph}-4\right)(\mathbf{1 0}): 3120$ w, 3034 w, 2964 m, 2927 m, 2869 m, 2584 m, 1735 w, 1597 m, 1517 sh, 1505 m, 1488 m, 1449 sh, 1401 m, 1387 m, 1365 m, 1293 w, 1277 sh, 1266 s, 1257 sh, 1195 s, 1173 m, 1162 w, 1107 m, 1083 m, 1070 m, 1051 s, 1029 w, 1022 w, 1004 w, 923 s, 902 s, 888 s, 837 m, 824 w, 800 m, 784 m, 759 s, 740 m, 731 m, 714 sh, 689 m, 646 w, 621 m, 615 sh, 489 w, 476 w, 425 w, $413 \mathrm{w} \mathrm{cm}^{-1}$.

$\mathrm{Tp}^{\mathrm{iPr}} \mathrm{MoO}_{2}\left(\mathrm{OC}_{6} \mathrm{H}_{4}{ }^{\mathrm{t}} \mathrm{Bu}-4\right)$ (11): 2966 s, 2927 m, 2868 m, 2580 m, 1889 m, 1507 s, 1459 m, 1403 m, 1387 m, 1363 m, 1294 w, 1255 m, 1195 m, 1179 m, 1106 w, 1082 m, 1070 m, 1050 s, 1020 w, 927 s, 904 s, 832 m, 799m, 780 m, 731 m, 712 w, 628 w, 552 m, 480 w, $442 \mathrm{w} \mathrm{cm}^{-1}$.

$\mathrm{Tp}^{\mathrm{iPr}} \mathrm{MoO}_{2}\left(\mathrm{OC}_{6} \mathrm{H}_{4}{ }^{\mathrm{B}} \mathrm{Bu}-4\right)$ (12): 3448 br, 2969 m, 2926 m, 2871 m, 2507 m, 1502 s, 1458 w, 1401 m, 1385 m, 1364 m, 1292 w, 1242 m, 1201 m, 1169 w, 1082 m, 1071 m, 1047 m, 930 m, 898 s, 876 m, 832 m, 798 m, 787 m, 772 m, 733 m, 580 w, 550 w cm'.

\section{Oxo(phosphine oxide)-Mo(IV) Complexes}

$\mathrm{Tp}^{\mathrm{iPr}} \mathrm{MoO}\left(\mathrm{OC}_{6} \mathrm{H}_{4}{ }^{\mathrm{t}} \mathrm{Bu}-2\right)\left(\mathrm{OPEt}_{3}\right): 3435$ br, 2965 s, $2867 \mathrm{~m}, 2488 \mathrm{~m}, 2477 \mathrm{~m}, 1589 \mathrm{w}, 1508$ s, 1478 m, 1438 m, 1401 m, 1385 m, 1364 m, 1291 m, 1255 m, 1241 w, 1194 s, 1122 s, 1070 s, 948 m, 929 w, 902 w, 866 w, 823 m, 793 w, 774 s, 746 m, 733 s, 632 m, 604 m, $450 \mathrm{w} \mathrm{cm}^{-1}$.

$\mathrm{Tp}^{\mathrm{iPr}} \mathrm{MoO}\left(\mathrm{OC}_{10} \mathrm{H}_{7}-\alpha\right)\left(\mathrm{OPEt}_{3}\right): 3448$ br, $3051 \mathrm{w}, 2968$ s, 2946 m, 2924 m, 2868 m, 2477 m, 2446 m, 1568 s, 1508 s, 1456 m, 1395 s, 1387 sh w, 1363 m, 1324 m, 1282 s, 1266 sh w, 1237 m, 1193 s, 1102 s, 1072 w, 1046 s, 1014 w, 948 m, 903 w, 892 w, 858 w, 794 w, 768 s, 734 s, 652 w, 628 w, 608 w, 583 w, 563 w, 506 w, 462 w, 477 w cm$^{-1}$.

$\mathrm{Tp}^{\mathrm{iPr}} \mathrm{MoO}\left(\mathrm{OC}_{6} \mathrm{H}_{4} \mathrm{Ph}-2\right)\left(\mathrm{OPEt}_{3}\right): 3433$ br, 3061 w, 2970 m, 2948 m, 2926 m, 2868 m, 2476 m, 2445 m, 1585 m, 1509 m, 1497 m, 1472 s, 1429 m, 1402 m, 1385 m, 1363 m, 1273 s, 1257 m, 1193 s, 1155 w, 1098 br m, 1075 w, 1043 s, 950 m, 906 w, 793 w, 773 m, 755 w, $735 \mathrm{~s}, 698 \mathrm{~m}, 632 \mathrm{w}, 617 \mathrm{w}, 603 \mathrm{~m}, 471 \mathrm{w} \mathrm{cm}^{-1}$.

$\mathrm{Tp}^{\mathrm{iPr}} \mathrm{MoO}\left(\mathrm{OC}_{6} \mathrm{H}_{4} \mathrm{Ph}-3\right)\left(\mathrm{OPEt}_{3}\right): 3434$ br, 2968 m, 2945 m, 2868 m, 2477 m, $2451 \mathrm{~m}, 1586$ m, 1560 m, 1508 m, 1473 s, 1411 m, 1402 m, 1384 m, 1364 m, 1309 m, 1302 m, 1263 m, 1217 m, 1194 s, 1161 m, 1087 s, 1043 s, 993 m, 949 s, 902 m, 869 m, 783 m, 777 m, 757 s, $735 \mathrm{~s}, 701 \mathrm{~m}, 616 \mathrm{~m}, 596 \mathrm{~m}, 466 \mathrm{w} \mathrm{cm}^{-1}$. 
$\mathrm{Tp}^{\mathrm{iPr}} \mathrm{MoO}\left(\mathrm{OC}_{10} \mathrm{H}_{7}-\beta\right)\left(\mathrm{OPEt}_{3}\right): 3432$ br, 3044 w, 2969 s, 2946 m, 2926 m, $2867 \mathrm{~m}, 2478$ m, 2447 m, 1619 s, 1590 s, 1565 w, 1509 s, 1498 sh w, 1464 m, 1435 w, 1402 w, 1384 m, 1365 m, 1339 m, 1272 s, 1260 sh w, 1220 m, 1194 s, 1177 m, 1101 s, 1079 sh w, 1045 s, 1014 w, 946 m, 913 m, 873 w, 848 m, 795 w, 772 m, 750 sh w, 733 s, 629 w, 621 w, 564 $\mathrm{w}, 543 \mathrm{w}, 508 \mathrm{w}, 474 \mathrm{w} \mathrm{cm}^{-1}$.

$\mathrm{Tp}^{\mathrm{iPr}} \mathrm{MoO}\left(\mathrm{OC}_{6} \mathrm{H}_{4} \mathrm{Ph}-4\right)\left(\mathrm{OPEt}_{3}\right): 3435$ br, 3203 w, 2962 s, 2927 m, 2869 m, 2449 m, 1595 m, 1512 s, 1480 s, 1458 w, 1403 w, 1385 m, 1364 m, 1286 s, 1273 s, 1190 s, 1164 m, 1103 s, 1070 w, 1046 s, 945 m, 903 w, 857 w, 840 w, 795 w, 777 m, 766 m, 734 s, 721 w, 698 w, $631 \mathrm{w}, 597 \mathrm{~m}, 478 \mathrm{w} \mathrm{cm}^{-1}$.

$\mathrm{Tp}^{\mathrm{iPr}} \mathrm{MoO}\left(\mathrm{OC}_{6} \mathrm{H}_{4}{ }^{\mathrm{t}} \mathrm{Bu}-4\right)\left(\mathrm{OPEt}_{3}\right): 3437 \mathrm{br}, 2966 \mathrm{~s}, 2925 \mathrm{~m}, 2867 \mathrm{~m}, 2452 \mathrm{~m}, 1601 \mathrm{w}, 1506$ s, 1459 m, 1401 m, 1385 m, 1363 m, 1281 m, 1264 m, 1195 s, 1173 w, 1083 s, 1044 s, 950 m, 902 w, 864 w, 833 m, 794 w, 772 m, 734 s, 690 w, 631 m, 539 w cm'$\mathrm{Tp}^{\mathrm{iPr}} \mathrm{MoO}\left(\mathrm{OC}_{6} \mathrm{H}_{4}{ }^{\mathrm{s}} \mathrm{Bu}-4\right)\left(\mathrm{OPEt}_{3}\right): 3435 \mathrm{~m} \mathrm{br}, 2966 \mathrm{~s}, 2927 \mathrm{~m}, 2869 \mathrm{~m}, 2473 \mathrm{~m}, 2446 \mathrm{~m}$, 1601 m, 1501 s, 1458 m, 1402 m, 1385 m, 1364 m, 1275 s, 1265 s, 1239 m, 1191 s, 1168 m, 1082 s, 1042 s, 950 s, 902 w, 864 m, 852 m, 831 m, 795 m, 771 s, 733 s, 718 m, 628 w, $551 \mathrm{w}, 467 \mathrm{w}, 426 \mathrm{w} \mathrm{cm}^{-1}$.

${ }^{\text {a }}$ For IR data for $\mathrm{Tp}^{\mathrm{iPr}} \mathrm{MoO}(\mathrm{OPh})\left(\mathrm{OPEt}_{3}\right)$, see Smith et al. J. Am. Chem. Soc. 2000, 122, 9298 and for the $\mathrm{X}=\mathrm{OC}_{6} \mathrm{H}_{4}{ }^{\mathrm{B}} \mathrm{Bu}-2, \mathrm{OC}_{6} \mathrm{H}_{4}{ }^{\mathrm{B}} \mathrm{Bu}-3$ and $\mathrm{OC}_{6} \mathrm{H}_{3}{ }^{\mathrm{t}} \mathrm{Bu}_{2}-3,5$ derivatives, see Millar et al. Chem. Eur. J. 2005, in press. 